\title{
GMR \\ Detection of Klinefelter syndrome by G-banding analysis combined with primed in situ labeling technique
}

\author{
J.-L. Gao ${ }^{1,2}$, X.-P. Ding ${ }^{1}$, D.-S. Liu ${ }^{1}$ and Y.-J. Zhu ${ }^{1}$ \\ ${ }^{1}$ Key Laboratory of Bio-Resources and Eco-Environment, \\ Ministry of Education, Institute of Medical Genetics, \\ Sichuan University, Chengdu, China \\ ${ }^{2}$ Chengdu Blood Center, Chengdu, China \\ Corresponding author: X.-P. Ding \\ E-mail: Brainding@scu.edu.cn
}

Genet. Mol. Res. 10 (4): 4026-4033 (2011)

Received September 28, 2011

Accepted November 16, 2011

Published December 21, 2011

DOI http://dx.doi.org/10.4238/2011.December.21.6

\begin{abstract}
Primed in situ labeling (PRINS) technique is an alternative to in situ hybridization for rapid chromosome screening. We employed triple-color PRINS technique to detect chromosomal abnormalities in Klinefelter syndrome patients diagnosed by G-banding karyotype analysis. Among 1034 infertile male patients, 134 were found to be cytogenetically abnormal, including 70 with chromosomal number abnormalities and 64 with chromosomal structure abnormalities. Among these cytogenetically abnormal patients, 56 were diagnosed as having Klinefelter syndrome. PRINS technique was used on cultured lymphocyte metaphase cells of the Klinefelter syndrome patients; the same result was obtained with G-banding karyotype analysis. PRINS proved to be a rapid and reliable method to detect numerical chromosome abnormalities in peripheral blood lymphocytes in metaphase.
\end{abstract}

Key words: G-banding karyotype analysis; Primed in situ labeling; Chromosome abnormalities; Klinefelter syndrome 


\section{INTRODUCTION}

Chromosome abnormality is one of the main factors leading to certain human genetic diseases and infertility as well. Related investigation (Bhasin et al., 1994) has demonstrated that infertility due to genetic factors accounts for $30 \%$ of overall infertility. Klinefelter disease is also known as congenital failure of testicular development. In this condition, chromosomes do not separate in reproductive cells during mitosis and meiosis. The prevalence rate of Klinefelter disease is 1.18 per 1000 or about $10 \%$ of overall male infertility (Medica et al., 2005). Primed in situ (PRINS) labeling is a novel molecular cytogenetic technique that combines the high sensitivity of polymerase chain reaction (PCR) with the cellular or chromosome localization of fluorescent signals provided by in situ hybridization (Koch et al., 1989). G-banding (Giemsa) is the conventional means of staining chromosomes to reveal their banding patterns (Barch, 1991). To explore a rapid and reliable chromosome detection method, we used G-banding analysis combined with the PRINS labeling technique to analyze the chromosomes in metaphases of cultured lymphocytes isolated from Klinefelter syndrome patients.

\section{MATERIAL AND METHODS}

\section{Sample collection for G-banding analysis}

A total of 1034 male infertility patients, seen at the professional reproduction hospital of Sichuan Reproductive Health Research Center between October 2007 and March 2010, aged 24-44 years (mean age of 30.35 \pm 4.56 ), were enrolled in this study. Three milliliters of heparin-anticoagulated peripheral blood was collected. The infertility criteria were defined as male patient with no children and meeting the following conditions; married for over 2 years; have not taken any contraceptive measure; physical examination of wife was normal.

\section{Sample collection for PRINS analysis}

The positive blood samples identified as Klinefelter syndrome by G-banding analysis were used for comparison with the PRINS detection results.

\section{Equipment}

The instruments used were a Peltier Thermal Cycler MG48G in situ reaction module, Olympus BX51 fluorescence microscopy system (Olympus Corporation, Japan), and Axioplan-2 imaging fluorescence microscope.

\section{Reagent}

\section{G-banding analysis reagents}

The reagents included: peripheral blood medium; $100 \mu \mathrm{g} / \mathrm{mL}$ colchicine; hypotonic solution; fixative solution, methanol:acetic acid (3:1); Giemsa staining, and $0.02 \%$ bovine trypsin. 


\section{PRINS reagents}

The reagents included denaturing solution: $70 \%$ deionized formamide/2X SSC solution; alcohol gradient solutions $(75,85$, and $100 \%)$, dNTP $(\mathrm{N}=\mathrm{A} / \mathrm{C} / \mathrm{G}$; Promega Corporation, USA), dTTP (Promega Corporation), dUTP-rhodamine (Roche Applied Science, USA), dUTP-digoxigenin (Roche Applied Science), $20 \mu \mathrm{g} / \mathrm{mL}$ anti-digoxigenin-FITC (Roche Applied Science); FITC fluorescence enhancer (Roche Applied Science); 1\% bovine serum solution (BSA), TaqDNA polymerase, Taq buffer (PCR buffer); Taqstart antibody (Takara Bio Inc., Japan), $10 \mu \mathrm{g} / \mathrm{mL}$ avidin-rhodamine (Roche Applied Science), TSATM Biotin System (PerkinElmer Inc., USA), fluorescence re-staining solution, $3 \mu \mathrm{g} / \mathrm{mL}$ DAPI (Sigma Corporation, USA); washing buffer (0.4X SSC/0.3\% NP-40 solution); NE solution ( $500 \mathrm{mM} \mathrm{NaCl}, 50$ mM EDTA, pH 8.0); Antifade solution (Applygen Technologies Inc., China); 1 mg/mL DAPI stock solution made with deionized water, diluted 1:300 with Antifade solution.

\section{G-banding analysis}

In the culture and pre-treatment procedure for preparing metaphases of peripheral blood lymphocytes (Ding, 2002), $0.5 \mathrm{~mL}$ blood was added to $5 \mathrm{~mL}$ culture medium and mixed, and the culture put in a $37^{\circ} \mathrm{C}$ incubator. The cultures were vibrated once or twice daily, and 1 $\mathrm{mL} 10 \mu \mathrm{g} / \mathrm{mL}$ colchicine was added after $72 \mathrm{~h}$. After mixing and continued culture for 4-6 h, the cells were collected. Hypotonic solution was added to swell the leukocytes, to disperse the chromosomes and to lyse red cells. After centrifugation and discarding the supernatant, the cells were treated three times with fixing solution, and the lymphocyte cell suspension was dropped on slides. The slides were flamed and air-fixed and banded, and the karyotype was analyzed with an automatic chromosome analyzer. A total of 30 metaphases were evaluated for each sample. Fifty metaphases were examined for abnormal karyotype, of which 10 were analyzed. The final analysis was conducted based on the International System for Human Cytogenetic Nomenclature.

\section{PRINS procedure}

Primers were synthesized by Invitrogen Corporation in China: primer sequence for chromosome 18: 5'-ATGTGTGTCCTCAACTAAAG-3'; primer sequence for chromosome X: 5'-GTTCAGCTCTGTGAGTGAAA-3'; primer sequence for chromosome Y: 5'-TCCATTCGATTCCATTTTTTTCGAGAA-3'; reaction labels were added consequently as digoxigenin-biotin-digoxigenin, corresponding to chromosome $18, \mathrm{X}$, and $\mathrm{Y}$, respectively.

According to the PRINS detection procedure (Liu and Ding, 2007), slides of lymphocyte metaphases were treated within 1-2 days after they were prepared. Denatured slides were put on a Peltier Thermal Cycler MG48G in situ reaction module at $66^{\circ} \mathrm{C}$ for annealing. When the slides reached primer-specific annealing temperature, reaction mixtures were added and preheated to $70^{\circ} \mathrm{C}$. The slides were covered with $22 \times 22 \mathrm{~mm}$ coverslips and sealed with mounting medium. Each primer was annealed at $66^{\circ} \mathrm{C}$ for $7 \mathrm{~min}$ and extended at $72^{\circ} \mathrm{C}$ for $10 \mathrm{~min}$; the slides were washed with $1 \mathrm{X}$ PBS between PRINS reactions to remove the previous reaction mixture. After completion of the reaction, $\mathrm{NE}$ solution at $72^{\circ} \mathrm{C}$ and 
$0.4 \mathrm{X} \mathrm{SSC} / 0.3 \% \mathrm{NP}-40$ solutions at $50^{\circ} \mathrm{C}$ were added, and the slides subsequently washed for $5 \mathrm{~min}$ to stop the extension reaction. Digoxigenin-11-dUTP-antidig-FITC indirect labeling method was employed to obtain the fluorescence signal. In a dark room, $20 \mu \mathrm{L} 20 \mu \mathrm{g} / \mathrm{mL}$ anti-digoxigenin-FITC fluorescence antibody solution was added. The PRINS fluorescence signal was obtained by a cell immunochemistry reaction; slides were sealed with sealing membrane and put in a humidified box at $37.5^{\circ} \mathrm{C}$, incubated for $30 \mathrm{~min}$, and washed with $1 \mathrm{X}$ PBS and $0.2 \%$ Tween 20 three times, 3 min each time; finally, the slides were re-stained with $3 \mu \mathrm{g} / \mathrm{mL}$ DAPI/Antifade solution.

Fluorescence signal was detected with the following systems: 1) Axioplan-2 imaging fluorescence microscopy system; 2) Olympus BX51 fluorescence microscopy system, Olympus UPlan FI 100X/1.30 Oil $\infty / 0.17 \mathrm{C} 1$ objective lens. The two systems were equipped with DAPI/FITC/rhodamine passband filter. Samples were taken by AxioCam Camera module and COHU Cooled CCD Camera; ISIS 5 (Meta Systems Company, Germany) and Video TestFISH 4.0 (Video TesT Ltd., Russia) were used for photography and analysis.

In this study, the PRINS technique was used to detect the chromosome sample of Klinefelter syndrome patients. Fifty metaphase nuclei were recorded for each slide. The recorded metaphase nuclei demonstrated clear fluorescence signals for correct chromosome number and target location. Overlapping or unclear signals in metaphase nuclei were not counted.

\section{RESULTS}

\section{G-banding analysis}

Among the 1034 male infertility patients studied, 134 (12.96\%) cases of karyotype abnormality were identified (Table 1). Of the 70 patients with chromosomal number abnormality, 56 (41.7\%) had Klinefelter syndrome (Figure 1), 4 cases had a number abnormality caused by chromosome translocation, and 1 case had 46,XY/47,XXY chromosome chimerism. The remaining $64(47.77 \%)$ cases had a chromosomal structure abnormality, 106 $(79.10 \%)$ cases with sex chromosome abnormality, and 28 (20.89\%) cases with autosomal abnormality. Karyotype abnormalities of different chromosome distribution are shown in Table 1.

\section{PRINS labeling of chromosomes of Klinefelter syndrome patients}

Fifty-six Klinefelter syndrome patients were identified by karyotype analysis. The blood sample was collected and chromosome spreads were made, after which PRINS detection was carried out. All samples were successfully labeled, with the exception of one case with $46, \mathrm{XY} / 47, \mathrm{XXY}$, for some unknown reason. Fluorescence signal matched the chromosome number change. Cell labeling results are presented in Figure 2, where the metaphase nuclei, specific chromosome target (chromosome 18, centromere region of X-chromosome, end of long arm of Y-chromosome), were stained with specific fluorescence signals (yellow, red and green). The $\mathrm{X}$-chromosome number abnormality result corresponded to that of G-banding analysis (red fluorescence signal), in which there were two red fluorescence signal (a, b). The correspondence suggested the accuracy of the PRINS method. 
Table 1. Distribution of abnormal karyotypes.

\begin{tabular}{|c|c|c|c|}
\hline Group & Karyotype & Case $(\mathrm{N})$ & Rate $(\%)$ \\
\hline \multirow[t]{9}{*}{ Sex chromosome, abnormal } & $47, \mathrm{XXY}$ & 55 & 5.32 \\
\hline & $46, X Y, X Y, y$ & 37 & 3.58 \\
\hline & $46, X X$ & 4 & 0.39 \\
\hline & $46, X Y, X Y, Y$ & 3 & 0.29 \\
\hline & $45, \mathrm{XO}$, delY & 3 & 0.29 \\
\hline & $46, \mathrm{XY} / 47, \mathrm{XXY}$ & 1 & 0.10 \\
\hline & 46,XY,delYp/45,XO,delY & 1 & 0.10 \\
\hline & $46, \mathrm{XY}, \mathrm{delYq}$ & 1 & 0.10 \\
\hline & $46, \mathrm{XY}, \mathrm{Yp}++$ & 1 & 0.10 \\
\hline \multirow[t]{14}{*}{ Autosome, abnormal } & $45, \mathrm{XY}, \mathrm{t}(13 ; 14)$ & 6 & 0.58 \\
\hline & $46, \mathrm{XY}, \mathrm{t}(1 ; 16)$ & 1 & 0.10 \\
\hline & $46, \mathrm{XY}, \mathrm{t}(1 ; 7)$ & 1 & 0.10 \\
\hline & $46, \mathrm{XY}, \mathrm{t}(2: 21)$ & 1 & 0.10 \\
\hline & $46, X Y, t(3 ; 4)$ & 1 & 0.10 \\
\hline & $45, \mathrm{XY}, \mathrm{t}(13 ; 18)$ & 1 & 0.10 \\
\hline & $45, \mathrm{XY}, \mathrm{t}(14 ; 15)$ & 1 & 0.10 \\
\hline & $45, \mathrm{XY},-22,-22,+\operatorname{rob}(22 ; 22)$ & 1 & 0.10 \\
\hline & $46, \mathrm{XY}, 15 \mathrm{ps}^{+}$ & 4 & 0.39 \\
\hline & $46, X Y, 21 \mathrm{ps}^{+}$ & 4 & 0.39 \\
\hline & $46, X Y, 13 \mathrm{ps}^{+}$ & 2 & 0.19 \\
\hline & $46, \mathrm{XY}, 14 \mathrm{ps}^{+}$ & 2 & 0.19 \\
\hline & $46, \mathrm{XY}, 22 \mathrm{ps}^{+}$ & 2 & 0.19 \\
\hline & $46, \mathrm{XY}, 1 \mathrm{qh}^{+}$ & 1 & 0.10 \\
\hline Total & & 134 & 12.96 \\
\hline
\end{tabular}

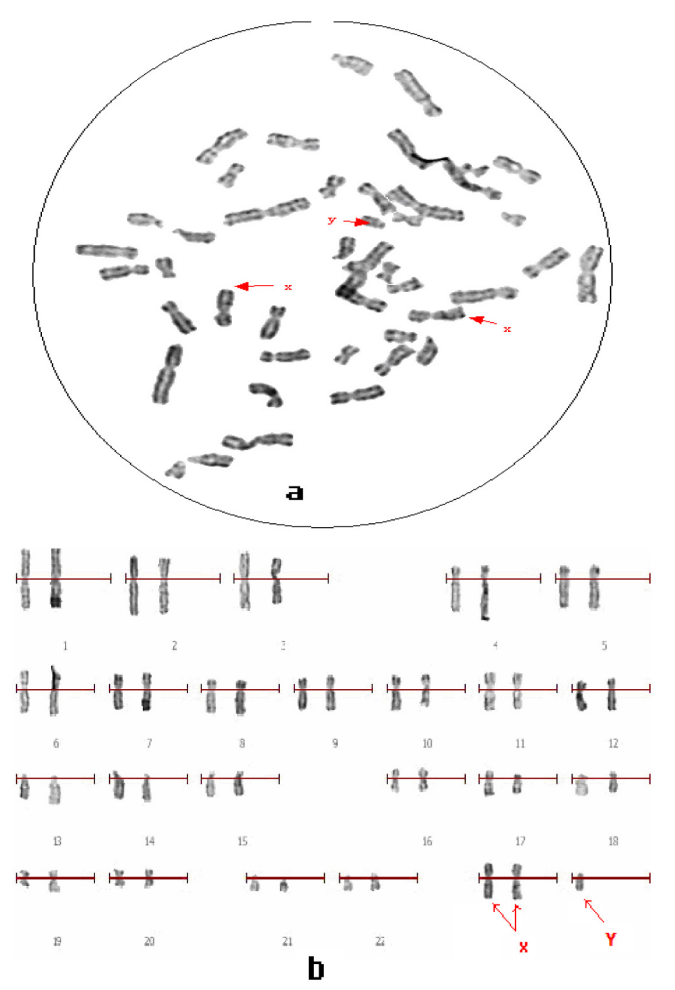

Figure 1. a. Mapping under microscopy or b. by software analysis in G-banding karyotyping of Klinefelter syndrome. 


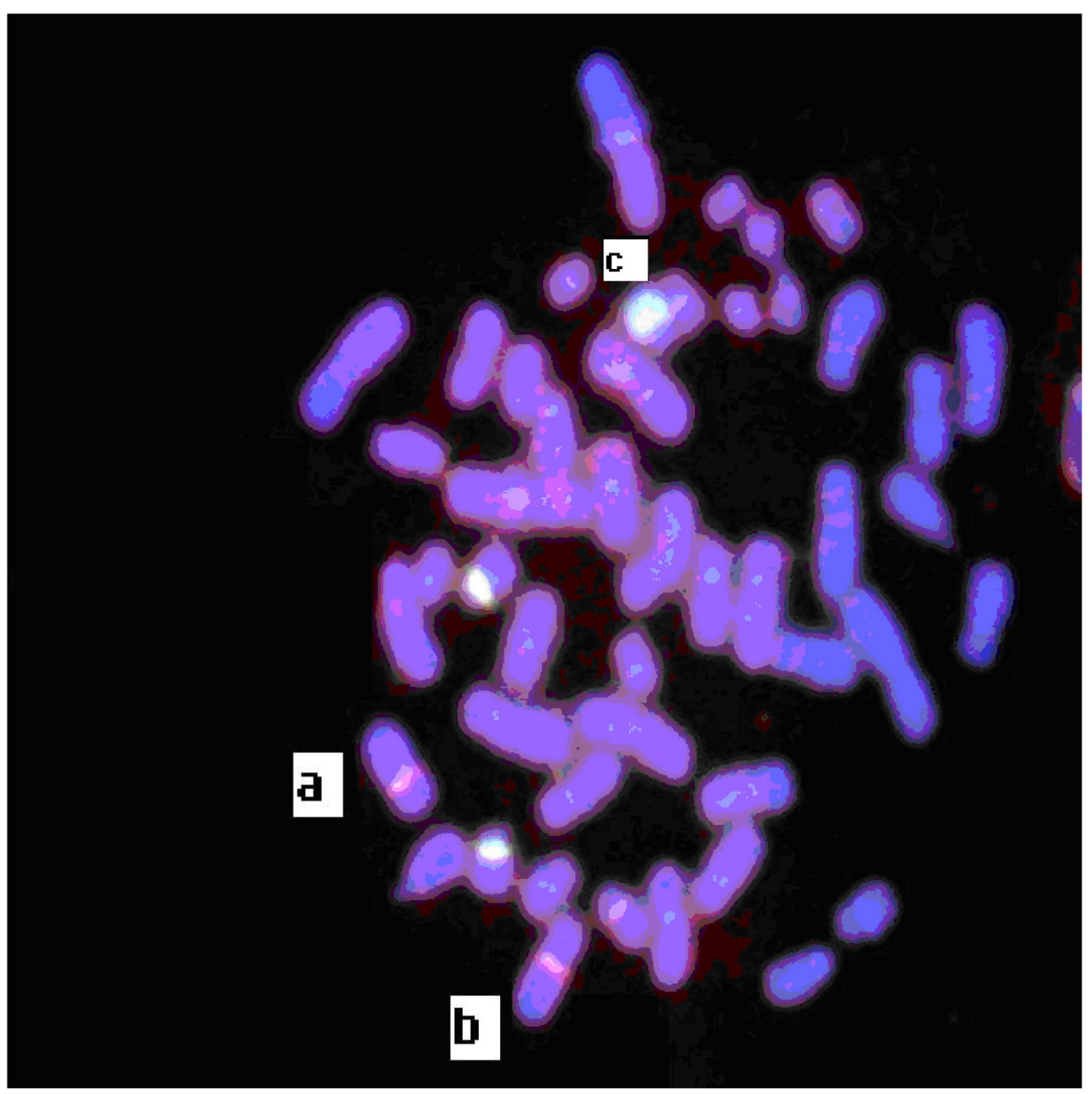

Figure 2. Triple-color PRINS labeling of peripheral lymphocyte metaphases from Klinefelter syndrome patients. Chromosome 18 is marked in yellow (two signals), X-chromosome in red (a and b, two signals), Y-chromosome in green $(c)$.

\section{DISCUSSION}

G-banding analysis is a reliable and inexpensive method for standard karyotype analysis. It has been widely used in the detection of chromosomal number abnormality and chromosome deletion and translocation.

In the infertility patients enrolled in this study, $13 \%$ patients demonstrated chromosomal number or structure abnormality. Van Assche et al. (1996) conducted a cell genetic study in 694 male infertility patients and discovered that the chromosome abnormality rate in infertility males was eight times more than that of normal males. Karyotype abnormality could 
be an abnormality in the structure of the sex chromosome or autosome or in the chromosomal number. One study (Maduro and Lamb, 2002) reported that regular chromosome abnormality accounts for $10 \%$ of overall chromosome abnormality. The ratio detected in this study was $20.89 \%$ (Table 1), in which there were 56 cases with Klinefelter syndrome, $41.7 \%$ of overall abnormality. Generally, G-banding analysis is able to meet karyotyping requirements for the detection of chromosome number abnormality and deletion. In this study, we mainly chose fresh samples for slides and appropriate digestion time. The working solution was prepared just before use. Bovine trypsin was used during digestion to extend chromosomes so that bands would appear clearer, consequently providing enhanced resolution. The reduced experiment time allows more accurate allocation of chromosome broken ends and improves reliability of the study and diagnosis (Gao et al., 2006).

Although G-banding analysis has greatly improved over time and it has been widely used, it is still difficult to allocate and detect chromosomal microdeletions accurately. The PRINS technique was developed by Koch et al., in 1989, and has been widely used for chromosome DNA in situ detection. The tyrosinamide signal amplification (TSA) system has been applied in in situ hybridization (Speel et al., 1997), which obviously enhances the sensitivity $\geq 100$ times without compromising solution and background to make the PRINS more feasible for single sequence detection. Tharapel and Wachtel (2006) used PRINS to detect a single gene. Earlier research on three-color PRINS blocked by ddNTP has demonstrated the improved detection flow in less time and cost without sacrificing labeling effect (Yan et al., 2001). It could be used for successful labeling of more than three target sequences at a time with different colors.

In this study, we labeled the chromosomes of 56 Klinefelter syndrome patients identified by G-banding analysis, where one case with $46, \mathrm{XY} / 47, \mathrm{XXY}$ was not labeled successfully for no valid reason.

In the current study, we amended the traditional PRINS technique and obtained a superior outcome for fluorescence signal and chromosome target allocation (Figure 2).

\section{Important amendments to the traditional PRINS}

We designed four identical primers and used the $\mathrm{TSA}^{\mathrm{TM}}$ Biotin System to enhance fluorescence intensity so as to make the corresponding detection system easier. The use of too many primers may cause pairing of primers themselves and require more complex primer designing. TaqStart antibody used in the reaction system could prevent the formation of primer dimers and nonspecific extension. The $0.4 \mathrm{X} \mathrm{SSC} / 0.3 \% \mathrm{NP}-40$ solution could produce a low background and improve signal contrast. We set different temperatures $\left(66^{\circ}, 72^{\circ} \mathrm{C}\right)$ for annealing and extension reactions of each specific primer to improve reaction specificity. By and large, the denaturing temperature was supposed to be maintained at $92^{\circ}-95^{\circ} \mathrm{C}$ (Therkelsen et al., 1995). By repeating the processes such as annealing, extension, and washing several times, cell and chromosome scaffolding will demonstrate obvious damage and final background will be high, which has a major influence on labeling rate in the PRINS reaction.

In contrast to other techniques, PRINS is rapid, reliable, sensitive and inexpensive, and because of these merits, it is an important analytical method used in the clinical diagnosis of genetic diseases and to study chromosome structure. The combination of a traditional genetic technique, G-banding analysis, and highly developed molecular genetic techniques is 
vital in chromosome disease diagnosis. As the advances of PRINS technique are becoming more accessible, it has been widely used in the medical genetic research field for single gene sequence detection and location, chromosome profile, banding, DNA sequence analysis, detection of carcinoma genes and anti-tumor mutations, etc.

\section{ACKNOWLEDGMENTS}

We extend our sincere thanks to Dr. Peter Pothula, BioQuest, for his valuable editorial guidance in the generation of this report.

\section{REFERENCES}

Barch MJ (1991). The ACT Cytogenetics Laboratory Manual. 2nd edn. Raven Press, New York.

Bhasin S, de Kretser DM and Baker HW (1994). Clinical review 64: Pathophysiology and natural history of male infertility. J. Clin. Endocrinol. Metab. 79: 1525-1529.

Ding X-P (2002). Modern Clinical Molecular Genetic and Cytogenetic Techniques. 1st edn. Sichuan University Press, Sichuan.

Gao LJ, Song F and Zhou L (2006). Effect of trypsin of different sources on G-banding analysis. J. Baotou Med. College 22: 77-78.

Koch JE, Kolvraa S, Petersen KB, Gregersen N, et al. (1989). Oligonucleotide-priming methods for the chromosomespecific labelling of alpha satellite DNA in situ. Chromosoma 98: 259-265.

Liu DS and Ding XP (2007). Triple-color primed in situ labeling protocol of human metaphase chromosomes. Zhonghua Yi Xue Yi Chuan Xue Za Zhi 24: 514-517.

Maduro MR and Lamb DJ (2002). Understanding new genetics of male infertility. J. Urol. 168: 2197-2205.

Medica I, Gligorievska N, Prenc M and Peterlin B (2005). Y microdeletions in the Istria county, Croatia. Asian J. Androl. 7: 213-216.

Speel EJ, Ramaekers FC and Hopman AH (1997). Sensitive multicolor fluorescence in situ hybridization using catalyzed reporter deposition (CARD) amplification. J. Histochem. Cytochem. 45: 1439-1446.

Tharapel AT and Wachtel SS (2006). PRINS for mapping single-copy genes. Methods Mol. Biol. 338: 59-67.

Therkelsen AJ, Nielsen A, Koch J, Hindkjaer J, et al. (1995). Staining of human telomeres with primed in situ labeling (PRINS). Cytogenet. Cell Genet. 68: 115-118.

Van Assche E, Bonduelle M, Tournaye H, Joris H, et al. (1996). Cytogenetics of infertile men. Hum. Reprod. 11 (Suppl 4): $1-24$.

Yan J, Bronsard M and Drouin R (2001). Creating a new color by omission of 3 end blocking step for simultaneous detection of different chromosomes in multi-PRINS technique. Chromosoma 109: 565-570. 